model of RA, RASF migrate through the blood stream to implanted cartilage independent of its chondrocyte viability and species background. The aim of this study was to analyse whether isolated RASF represent a homogenous migratory cell population.

Methods Healthy human cartilage was implanted subcutaneously into SCID mice together with $5 \times 10^{5}$ RASF (ipsilateral; I). At the contralateral flank, cartilage without RASF was implanted (contralateral; C). The animals were divided into four groups and 14 days after the first implantation the implants were in part removed and inserted into other animals. In group 1, the ipsilateral implant was replaced and transferred into group 2. Additionally, group 2 received a new contralateral implant. New contralateral implants were implanted into group 3. Group 4 served as control, no implant replacement took place. Seventy days after the first implantation all implants were removed and analysed histologically regarding cartilage invasion (inv) and perichondrocytic cartilage degradation (deg).

Results RASF invaded the ipsilaterally implanted cartilage (group 4: I inv: $2.3 \pm 0.5$; deg: $1.5 \pm 0.5$ ) and migrated to the contralaterally implanted cartilage and invaded it ( $\mathrm{C}$ inv: $1.8 \pm 0.9$; deg: $1.5 \pm 0.5)$. No invasion into the contralateral implant was seen after transfer of the ipsilateral cartilage into new animals (group 2: I inv: 2.6 \pm 0.5 ; deg: $1.8 \pm 0.4$; C inv: $0.3 \pm 0.3$; deg: $1.0 \pm 0.5)$. Cartilage destruction was observed when replacing the contralateral implant (group 3: I inv: $2.3 \pm 0.3$; deg: $1.5 \pm 0.8$; C inv: $1.5 \pm 0.5$; deg: $0.9 \pm 0.3$ ). After implantation of a new ipsilateral RASF-containing implant, similar invasion scores were observed at both sides (group 1: I inv: $2.2 \pm 0.5$; deg: $1.3 \pm 0.4$; C inv: $2.0 \pm 0.4$; deg: $1.2 \pm 0.5$ ).

Conclusion Implanted RASF of a patient represent a heterogeneous cell population. Subpopulations differ in their migratory behaviour. RASF migration takes place in the first 2 weeks. The remaining cells do not migrate but invasion of the directly coimplanted cartilage continues. Migrated RASF were detectable at the contralaterally implanted cartilage after 14 days. Nevertheless, cartilage destruction was not visible at this time point. As it is possible to reculture the RASF out of the implants, further analysis of the subpopulations will help to characterise the destructive potential of RA subpopulations showing differential migratory behaviour.

\section{A137 MIGRATING SYNOVIAL FIBROBLASTS: A HOMOGENOUS POPULATION?}

S Lefèvre, B Zimmermann, A Lehr, H Stürz, J Steinmeyer, S Rehart, U Müler-Ladner, E Neumann JLU Gießen/Kerckhoff-Klinik, Germany

\subsection{6/ard.2010.129643n}

Background Chronic inflammation and cartilage destruction are central features of rheumatoid arthritis (RA). RA synovial fibroblasts (SF) play a key role in cartilage destruction and in the spreading of the disease by migrating to and invading into cartilage. As demonstrated recently in the SCID mouse 